\title{
Value of Intraoperative Brainstem
}

\section{Auditory Evoked Potential}

Monitoring in Reducing the

\section{Auditory Morbidity Associated}

\section{with Microvascular Decompression}

of Cranial Nerves

\begin{abstract}
The present study was performed to determine whether the intraoperative monitoring of brainstem auditory evoked potentials (BAEPs) during microvascular decompression operations is effective in preventing profound hearing loss or deafness in the ipsilateral ear. The authors retrospectively compared the auditory morbidity of posterior fossa microvascular decompression surgery for the treatment of tic douloureux and hemifacial spasm before and after the introduction of routine intraoperative BAEP monitoring in 1984. Each patient underwent a similar procedure performed by the same surgeon. The two patient groups were comparable with regard to age, sex, and indications for surgery. Auditory morbidity did not decline with the increasing experience of the surgeon prior to $1984 ; 10(6.6 \%)$ of 152 primary operations (151 patients) in which monitoring was not performed were followed by a profound ipsilateral hearing loss or deafness. In the monitored group, none of 109 operations (104 patients) caused profound hearing loss or deafness. This significant decline in auditory morbidity is attributed by the authors to the use of intraoperative BAEP monitoring, which allows the surgeon to alter the operation in response to degradations in the wave patterns. Based on our experience and that of others, we believe that intraoperative BAEP monitoring is of value in reducing the auditory morbidity of posterior fossa microvascular decompression surgery.
\end{abstract}

Monitoring of brainstem auditory evoked potentials (BAEPs) during posterior fossa operations has become routine practice in many medical centers, including ours. ${ }^{1-14}$ The continuous intraoperative recording of BAEPs permits neurophysiologic assessment of auditory and brainstem function that is not otherwise possible in the anesthetized patient. Changes in waveform are thought to signal an abnormality along the auditory pathways and provide a warning to the surgeon, who can then alter the operation such as by releasing cerebellar retraction, in the hope that permanent neural damage may thus be averted.

From December 1975 through June 1983 and January 1984 through June 1989, 261 microvascular decompression operations for the treatment of tic douloureux (191) or hemifacial spasm (71) were performed by the same surgeon (RHW) using the same criteria for patient selection. One of these operations involved the simultaneous treatment of ipsilateral tic douloureux and hemifacial spasm

Skull Base Surgery, Volume 1, Number 2, April 1991 Divisions of Neurosurgery and Neurology, Duke University Medical Center, Durham, North 
(tic convulsif). These operations were conducted without BAEP monitoring before $1984^{15}$ and with it beginning in January of that year. To determine whether such monitoring protected the auditory pathways, we retrospectively analyzed the evoked potential data and auditory outcome, and compared these between the two groups of patientsthose who did not have BAEP monitoring and those who did.

\section{METHODS}

\section{Patient Population}

The 151 nonmonitored patients in whom 152 primary operations (that is, first posterior fossa procedures) were performed for tic douloureux (104) or hemifacial spasm (48) were operated on either at the University of Pittsburgh (December 1975 through October 1976) or at the Duke University Medical Center (March 1977 through June 1983). ${ }^{15}$ One patient in this group was operated on two separate times for bilateral tic douloureux; she was counted as one patient and two operations. Another patient had one operation for tic convulsif and therefore was counted separately in the tic group and in the hemifacial spasm group as though two operations were done on two patients. The 109 monitored operations (104 patients) for tic douloureux (86) or hemifacial spasm (23) were performed at the Duke University Medical Center between January 1984 and June 1989.

The two groups of patients had similar characteristics. The mean age of the nonmonitored patients was 55.1 years and that of the monitored patients was 53.9 years. The male:female distribution for the operations done without monitoring was 54:98 and for those with monitoring was 47:62. The right:left distribution of the first group was 83:69 and of the second was 65:44.

\section{Operation}

The rationale for microvascular decompression surgery has been reviewed elsewhere. ${ }^{15-18}$ The patients operated on in the present series had either typical tic douloureux or hemifacial spasm and did not have a causative neoplasm in the posterior fossa. All were healthy enough to undergo the operation, and those with tic douloureux had become unresponsive to medical management. The criteria for patient selection for surgical treatment did not change appreciably during the period of this study.

A small unilateral retromastoid craniectomy was performed under general anesthesia and the dura mater was opened. Using a self-retaining retractor and standard microsurgical technique, the surgeon retracted the cerebellum, opened the arachnoid, and exposed the trigeminal or facial nerve at the brainstem. If the surgeon found compression of the nerve at this junction by an adjacent artery or large vein, he separated the vessel from the neural tissue and inserted a sculptured sponge of polyvinyl alcohol foam (Ivalon; Unipoint Laboratories, High Point, NC) to maintain the separation. If a small vein or veins were thought to be causing or adding to such compression, these were coagulated and divided. If no offending vessel was found in a patient with tic douloureux, the caudal half of the main sensory trigeminal root was sectioned.

In general, there were fewer complications associated with these operations as the surgeon gained experience. However, the auditory morbidity was not affected until intraoperative BAEP monitoring was begun. Of the first 76 nonmonitored operations, five were followed by a profound ipsilateral hearing deficit and three by a mild loss; the comparable figures for the second 76 nonmonitored operations were five and five.

\section{Intraoperative BAEP Monitoring}

Our technique for obtaining BAEPs intraoperatively has been described previously.8,10,12 Briefly, the ear on the side of the operation is stimulated by a 70 to $95 \mathrm{~dB}$ HL alternating polarity click stimulus at a rate of $31 / \mathrm{sec}$. Twochannel derivations using ipsilateral ear $(\mathrm{Ai})$ to vertex $(\mathrm{Cz})$ and contralateral ear $(\mathrm{Ac})$ to vertex $(\mathrm{Cz})$ are recorded. Routing filter settings are low-frequency filter of $150 \mathrm{~Hz}$ and high-frequency filter of $3000 \mathrm{~Hz}$. Each average contains 1000 to 4000 repetitions and can be completed in 1 to 2 minutes. Determination of wave $\mathrm{V}$ latency is usually possible within 10 to 20 seconds of the start of a new trial. The preoperative averaged baseline tracing as well as the preceding intraoperative averaged tracings are displayed serially to help in the assessment of any change in the recorded responses.

The primary criteria of change were the latency and form of wave $V$. Latency changes equal to or greater than $1.0 \mathrm{msec}$ or rapid changes greater than $0.1 \mathrm{msec} / \mathrm{min}$ were ordinarily considered indicators of neural compromise, and this information was communicated immediately to the surgeon. This usually resulted in an alteration of the surgical procedure, such as the repositioning or removal of the cerebellar retractor, until definite improvement in the BAEP response was noted (often as quickly as the next averaged tracing). It was interesting that typically the deterioration of the latency or form of wave $V$ occurred when the surgeon was unaware of anything amiss, such as excessive traction on the auditory nerve.

\section{Assessment of Auditory Function}

In both patient groups, the presence or absence of a postoperative auditory deficit was determined by history, clinical testing, and audiometry. All patients were ques- 
tioned about the status of their hearing prior to discharge from the hospital and at the time of their first follow-up visit 1 month later. Any complaint of decreased hearing on the operative side that was not present preoperatively was considered to be evidence of an auditory deficit. Many of these were mild, transient deficits related to fluid in the middle ear that subsequently resolved, but they were still counted in our study as a possible auditory complication.

The patients with a profound hearing loss or deafness were easy to identify by history and physical examination. Pre- and postsurgical audiometry was performed for 15 $(10 \%)$ of the 152 nonmonitored operations and for 79 (72\%) of the 109 monitored operations. Audiometric evaluation was performed in the evoked potential laboratory (nonacoustically shielded environment) with determination of pure-tone thresholds for 500, 1000, 2000, and 4000 $\mathrm{Hz}$. Bone conduction and speech threshold testing were not performed, and no comment can be made on the contribution of conductive versus sensorineural hearing loss in patients with an identified deficit.

Auditory outcomes were classified in three groups. Those patients with no deficit had no subjective auditory complaints and no loss more than $10 \mathrm{~dB}$ on audiometry. A mild deficit was one noted by the patient or one associated with a 15 to $30 \mathrm{~dB}$ loss on audiometry. A profound deficit represented a major or complete clinical loss of auditory function or an audiometric loss of more than $30 \mathrm{~dB}$. In practice, all patients with a greater than $30 \mathrm{~dB}$ loss had a profound and irreversible loss of functional hearing in that ear, presumably on the basis of mechanical injury or ischemia involving the acoustic nerve or cochlea.

\section{RESULTS}

A profound ipsilateral hearing loss occurred in 10 (6.6\%) of the 152 operations performed without BAEP monitoring. During the 109 subsequent operations performed with such monitoring, the intraoperative BAEP changes were as follows: in 68 there was less than $1 \mathrm{msec}$ change in wave $\mathrm{V}$ latency, in 26 there was more than a 1 msec change, in 12 there was a transient loss of wave $\mathrm{V}$, and in three there was a persistent loss. The postoperative hearing losses were all mild, occurring in three of the 68 operations with less than a $1 \mathrm{msec}$ change, in two of the 26 with more than a $1 \mathrm{msec}$ change, in three of the 12 with a transient wave loss, and in one of the three with a persistent wave loss. No monitored patient had a profound hearing loss, which represents a statistically significant difference when compared with the nonmonitored patients (Table 1).

\section{DISCUSSION}

Many groups now monitor BAEPs during operations in the posterior fossa, including microvascular decompression operations. 1-14 Prior to such monitoring (19711979), Jannetta and colleagues 1,16 at the University of Pittsburgh noted that profound hearing loss or deafness ipsilateral to the site of operation occurred in 16 of 229 (7\%) patients.

In 1981, that group reported a case in which intraoperative BAEP changes gave the surgeon sufficient warning to prevent a clinically evident hearing loss. ${ }^{1}$ The patient was being operated on for treatment of right hemifacial spasm. "Anesthesia and surgery were uneventful until retractors were placed on the cerebellum, and microvascular decompression of the seventh nerve was begun at 9:20 a.m. An increase in the poststimulus latency of peak $\mathrm{V}$ was noted 10 minutes later. No undue retractor pressure was apparent on visual inspection and the operation proceeded without alteration of retraction. Peak V latencies progressively increased until 10:16 a.m., when the BAEP past peak I was virtually obliterated. . . . The surgeon then repositioned the retractor and the BAEP complex reappeared, although still distorted, at 10:21 a.m. Recovery of the wave form continued during the remaining 45 minutes of anesthesia but was incomplete. The patient awoke in the operating room neurologically intact, relieved of her hemifacial spasm and with clinically normal hearing. . . . An audiogram done 4 days after surgery showed hearing to be the same as before surgery . . . in the right ear except for a slight decrease in hearing level at $2000 \mathrm{~Hz}$. The BAEP done 4 days after surgery showed normal latency for peak $\mathrm{V}$ but newly increased latencies for peaks II and III. . . . This case is of interest because virtually complete obliteration of the BAEP, apparently due to pressure from a retractor in the posterior fossa, was shown to be reversible."1

The predictive value of wave $\mathrm{V}$ changes is not absolute. As Watanabe et al ${ }^{13}$ have noted, postoperative hearing loss is possible despite the intraoperative preservation of wave V (two of their 68 cases), and postoperative hearing preservation may occur despite the intraoperative loss of wave V. Friedman and colleagues ${ }^{9}$ have empha-

Table 1. Audiologic Morbidity after Microvascular Decompression

\begin{tabular}{llccc}
\hline & & \multicolumn{3}{c}{ Auditory Outcome } \\
\cline { 2 - 5 } BAEP Monitoring & Number of Operations & No Deficit & Mild Deficit & Profound Deficit \\
\hline No & 152 & $134(88.2 \%)$ & $8(5.3 \%)$ & $10(6.6 \%)^{*}$ \\
Yes & 109 & $100(91.7 \%)$ & $9(8.3 \%)$ & $0^{*}$ \\
\hline
\end{tabular}


sized the latter point, noting that even in their four patients in whom wave $\mathrm{V}$ totally disappeared during cerebellar retraction, postoperative deafness did not occur. ${ }^{9}$ In this context, of our three patients who experienced a persistent loss of wave $\mathrm{V}$ intraoperatively, only one had a postoperative hearing deficit, and it was mild.

Even so, we believe that progressive BAEP changes during posterior fossa microvascular decompression operations do serve as a useful warning, permitting the surgeon to alter the operative procedure and preserve hearing. Our own experience, reported here, supports this view, as does the experience of the University of Pittsburgh group, who reported only two instances of significant hearing loss following 140 recent microvascular decompression operations, ${ }^{14}$ a $1.4 \%$ incidence compared with the previously mentioned 7\% incidence without BAEP monitoring.

It is of interest that the one recent Pittsburgh patient who had postoperative deafness was being operated on a second time for hemifacial spasm. ${ }^{14}$ Since the conclusion of patient acquisition for the present study, we have also had two patients who lost hearing during a second microvascular decompression operation for recurrent hemifacial spasm or tic douloureux, despite intraoperative BAEP monitoring. In both cases, wave $\mathrm{V}$ was lost abruptly during dissection around the previously placed Ivalon sponge. This maneuver seems especially hazardous in regard to auditory morbidity.

\section{REFERENCES}

1. Grundy BL, Lina A, Procopio PT, Jannetta PJ: Reversible evoked potential changes with retraction of the eighth cranial nerve. Anesth Analg 60:835-838, 1981

2. Allen A, Starr A, Nudleman K: Assessment of sensory function in the operating room utilizing cerebral evoked potentials: A study of fifty-six surgically anesthetized patients. Clin Neurosurg 28: 457-481, 1981

3. Raudzens PA: Intraoperative monitoring of evoked potentials. Ann NY Acad Sci 388:308-325, 1982

4. Raudzens PA, Shetter AG: Intraoperative monitoring of brain-stem auditory evoked potentials. J Neurosurg 57:341-348, 1982

5. Grundy BL, Jannetta PJ, Procopio PT, Lina A, Boston JR, Doyle E: Intraoperative monitoring of brain-stem auditory evoked potentials. J Neurosurg 57:674-681, 1982

6. Grundy BL, Procopio PT, Jannetta PJ, Lina A, Doyle E: Evoked potential changes produced by positioning for retromastoid craniectomy. Neurosurgery 10:766-770, 1982

7. Grundy BL: Monitoring of sensory evoked potentials during neurosurgical operations: Methods and applications. Neurosurgery 11:556-575, 1982

8. Piatt JH Jr, Radtke RA, Erwin CW: Limitations of brain stem auditory evoked potentials for intraoperative monitoring during a posterior fossa operation: Case report and technical note. Neurosurgery 16:818-821, 1985

9. Friedman WA, Kaplan BJ, Gravenstein D, Rhoton AL Jr: Intraoperative brain-stem auditory evoked potentials during posterior fossa microvascular decompression. J Neurosurg 62:552557, 1985

10. Radtke RA, Erwin CW: Intraoperative monitoring of auditory and brain-stem function. Neurol Clin 6:899-915, 1988

11. Møller AR: Evoked Potentials in Intraoperative Monitoring. Baltimore: Williams \& Wilkins, 1988

12. Radtke RA, Erwin CW, Wilkins RH: Intraoperative brainstem auditory evoked potentials: Significant decrease in postoperative morbidity. Neurology (Cleve) 39:187-191, 1989

13. Watanabe E, Schramm J, Strauss C, Fahlbusch R: Neurophysiologic monitoring in posterior fossa surgery: II. BAEP-waves I and $\mathrm{V}$ and preservation of hearing. Acta Neurochir (Wien) 98:118-128, 1989

14. Møller AR, Møller MB: Does intraoperative monitoring of auditory evoked potentials reduce incidence of hearing loss as a complication of microvascular decompression of cranial nerves? Neurosurgery 24:257-263, 1989

15. Piatt JH Jr, Wilkins RH: Treatment of tic douloureux and hemifacial spasm by posterior fossa exploration: Therapeutic implications of various neurovascular relationships. Neurosurgery 14 462-471, 1984

16. Jannetta PJ: Neurovascular compression in cranial nerve and systemic disease. Ann Surg 192:518-525, 1980

17. Wilkins RH: Neurovascular compression syndromes. Neurol Clin 3: 359-372, 1985

18. Wilkins RH: Surgical therapy of neuralgia: Vascular decompression procedures. Semin Neurol 8:280-285, 1988

\section{REVIEWER'S COMMENTS}

The work of Wilkins, Radtke and Erwin is of great interest. Indeed it statistically proves on a series of patients that the intraoperative BAEP monitoring brings a bigger security for a good maintenance of the auditory function in case of "microvascular decompression of cranial nerves." Indeed, as underlined by the authors, we have all of us experience of isolated cases with perioperative tracing damaged and good postoperative hearing or the inverse. This study is then very useful for surgeons using this kind of procedure.

M. Portmann, M.D. 